\title{
Effects of Cluster Network Structure to the Behavior of Enterprise Imitation
}

\author{
Lei Wang ${ }^{1, *}$ and Weimin Pan $^{2}$ \\ ${ }^{1}$ Department of management, Hangzhou Dianzi University, Hangzhou 310018, China \\ ${ }^{2}$ School of management, Hangzhou Dianzi University, Hangzhou 310018, China
}

\begin{abstract}
Since the 1990s, the research of cluster economy gradually becomes a topic of interest for academic economists and Management experts. In order to obtain the effects of cluster network structure on the behavior of enterprise imitation, based on neighbor effect, this paper builds dynamic model of a single enterprise income, then explores the propagation of corporate behavior under random network model, small world network model, scale-free network model. On the basis of neighbor average profit, this article makes a comparative analysis of the network structure imitation and then arrives at the cluster network structure's influence on the enterprise imitation.
\end{abstract}

Keywords: Enterprise imitation behavior, industrial clusters, neighbor effect, network structure.

\section{INTRODUCTION}

Industrial cluster forms a varied network structure, with different network structures having different characteristics and laws of propagation behavior. Barabási and Albert reports on the existence of a high degree of self-organization, characterizing the large scale properties of complex networks, Xiao Fan Wang and Guanrong Chen provide some introduction and insights into the new discipline of complex networks, M. E. J. Newman outlines some recent developments in the theory of social networks, particularly in the characterization and modeling of networks, in how networks change over time, and in the modeling of the spread of information or disease over networks. In this paper we provide that common network structure, such as random network structure, small-world network structure and scale-free networks structure, affects the behavior of enterprise imitation by different ways.

\section{THE ANALYSIS OF CHARACTERISTICS LAW OF BEHAVIOR SPREADING IN THREE DIFFERENT NETWORK STRUCTURES}

\subsection{A. The Law of Behavior Spreading in Random Net- work}

Random network, its network distribution is approximately subject to the Poisson distribution. It has two notable features: the set of nodes in the network and its affiliates with neighbor nodes have a significant randomness character; the distribution of nodes is approximately subject to the Poisson distribution.
In a random network, the average node sets the scale of the network, which means that most nodes have about the same number of links as the average node [1]. In the network, there are neither much higher nor much lower than the average link number in group, and with an infinite time, the majority of the number of edges in the network nodes tend to show convergence.

In this paper, the law of behavior spreading in random network are summarized as following points: the location chosen and its behavior spreading in random networks have a significant random character; Since the number of neighbors of each node in the network have little difference, therefore, with time, group behavior will show gradual convergence condition, despite the behavior of the network structure exhibiting a significant random character,

\subsection{B. The Law of Behavior Spreading in Small-world Network}

Small-world network is a network model with the character of small-world and high aggregation. People routinely claim that, global statistics notwithstanding, it is still a small world [2]. Generation rules of small-world network are as follows: This is a network with $\mathrm{N}$ nodes, where a single node and its distance from the closest neighbor and sub-neighbor connections connected to each other so as to form a ring with a random connection probability $\mathrm{p}$.

That is to say, the network has a high aggregation [4]. The node is closely related with the business of very close geographic proximity; the connection of one node with another are realized by the probability $\mathrm{P}$, and only one edge is allowed to be connected between any two nodes and any node cannot be connected with itself. In other words, there is also a small-world network randomness associated property.

Thus, in this article, the propagation rules of small-world networks are summarized in the two following points: There 
is close contact between neighboring nodes, strong overall coordination, and good promotion within clusters. Uniformity and randomness exists simultaneously. The behavior of corporation also spreads with rules (temporary or full convergence characteristics) and randomness (transient or continuous wave characteristics).

\subsection{The Law of Behavior Spreading in Scale-free Networks}

Scholars Barabasi, Albert and Jeong questioned the Poisson distribution network through a lot of research on networks and made supplement. They believe, in reality, there are many forms which meet the power-law distribution network. Power-law degree distribution, called scale free [3], a scale-free network is inhomogeneous in nature [4]. For example in the well-known World Wide Web, the study found, only a few well-known sites have a far more significant effect than the general level of large number of links, however, most of the pages only have a few links, that is, only a few nodes are connected with a large degree, while the vast majority of nodes are connected with only a small degree, this characteristic is called scale-free networks characteristics. Many large-scale complex networks are scale-free, that is, their connectivity distributions are in a power-law form that is independent of the network scale. In general, scale-free networks display an amazing robustness against accidental failures [5]. To better explain the principle of power-law distribution generated from networks, Barabbas and Albert further proposed scale-free network model, also known as the BA scale-free network model. A scale-free network, with two significantly different features from other network features, such as: Growth characteristics. The first network has a total of $0 x$ nodes, with time, new nodes will continually be introduced to the network, the new node will connect to the existing node in the network one by one; and priority connect features. That is, new entrants tend to choose nodes connected with a higher degree of "big" nodes, this phenomenon is similar to the management theory of "Matthew (Matthew Effect)". The Matthew Effect according to which the rich get richer and the poor get poorer is a principle in sociology and economics [6]. When selecting a new node connection, assuming that the probability $\pi$ (ki) that new node connected to node $i$, depends on the degree of node $i$ (where, $\mathrm{N}$ is the number of nodes in the network).

In short, BA scale-free networks have two important structural characteristics, one is the growth characteristics, namely the continually expanding of the network size; the other is the priority connectivity, new entrants tend to choose nodes connected with a higher degree of "big" node to connect. This is similar to enterprise imitation behavior in the cluster, both of which are derived from the characteristics of core business. Based on discussion above, the law of behavior spreading in scale-free networks can be summarized in the following two points: In the cluster, there are nodes with much higher than average degree of clustering, that is, leading enterprises, small degree nodes are significantly affected by big degree nodes; With the constantly changing tactics of large node behavior, group behavior presents the overall convergence or randomness (volatility).

\section{THE CONSTRUCTION OF DYNAMIC EN- TERPRISES INCOME MODEL BASED ON THE NEIGHBORHOOD EFFECT}

\subsection{A. The Standard of Neighbor Defying}

In clusters, there is a wide variety of links between corporations, including formal links and informal links. As to formal link, it can be connected by technology patents transfer and R \& D cooperation, as to informal link, it can be connected by material exchange and reverse R \& D of competing products. The informal contact brings a lot of indirect convenience for intra-cluster enterprises to imitate behavior. Estimating conditional dependencies based on the Local Markov Property specified by neighborhoods leads to a simple algorithmic strategy: estimate the neighborhood of each gene separately [7]. Porter (1998) defines industrial clusters as "geographic concentrations of competing, complementary, or interdependent firms" [8]. Combining related theoretical results and the field research of case clusters, the paper selected the "kinship" as standard of defining the neighbor in a network. It is based on this "kinship" that constitutes the link between enterprises in the cluster (or connection). Kinship is fascinating [9] the so-called kinship relationship, contains both kinship within three generations. Three generations as less direct, collateral consanguinity, also includes geopolitical or other relationships with a significant degree of discrimination such as old classmates, former colleagues, neighbor and so on.

\subsection{B. The Imitation Behavior Based on the Neighbor- hood Effect}

The existing interpretation about enterprise imitation behavior mechanisms in the cluster is focused on three perspectives, they are information cost saving, bounded rationality and its uneven distribution in the population and knowledge spillovers. Neighbor behavior exerts a significant influence on other neighbors' behavior [10]. What is different from above perspectives is that this paper regards "the neighborhood effect" as the core idea to interpret this imitation behavior. We tend to believe that because of the geographical proximity of industrial clusters creates a significant "neighborhood effect" characteristic, affecting the economics of direct investment [11], that is to say, neighborhood has a great impact on how individual corporate makes behavioral decisions in clusters. Based on analysis above, the article further puts forward a new view that whether individual corporate imitate the behavior of others depends on the earnings they received and the earning the neighbor received in the previous financial cycle under different strategic choices.

\subsection{The Model Building of Individual Corporate Dy- namic Income}

The function of individual income this paper sets is that

$S_{\mathrm{i}, \mathrm{j}}=N_{i-1} \times(1+x-\beta)$

Where:

Dynamic gain value $S_{i, j}$ is the single enterprise in the process of the game 
Ni-1 represents the original stock that a individual company received last round of the game, when $i=1, \mathrm{~N} 0$ is the original innovation earning value when company $\mathrm{j}$ is not involved in the game, to facilitate the study, we assume N0 = a (a is a constant greater than 0 )

i represents the number of the game;

$\mathrm{j}$ represents the node;

$\mathrm{x}$ represents the innovation earning coefficient that a single enterprise gained when it makes an innovation, Having referred to a lot of literature and carried out discussion about relevant theories, the paper sets a reasonable value to $\mathrm{x}$, that is $\mathrm{x}=0.3$.

Brepresents the technology spillover coefficient generated after a single enterprise $\mathrm{j}$ makes an innovation.

In addition, in the network which has a total of m nodes, this paper introduces $Y_{i, j}$ to distinguish different strategic choices that node $\mathrm{j}$ makes, if node $\mathrm{j}$ adopts innovative strategies during the $i$-th game, then $Y_{i, j}=1$; If node $j$ adopt imitative strategy during $i$-th game, then $Y_{i, j}=0$ Thus, coefficient of innovation, technology spillover coefficient values and individual corporate strategies to select, are further grouped into formula 2 and formula 3

$X= \begin{cases}0.3 & Y_{i, j}=1 \\ 0 & Y_{i, j}=0\end{cases}$

That is, when node $\mathrm{j}$ adopts innovation strategy during the $\mathrm{i}$-th game, $\mathrm{Y}_{\mathrm{i}, \mathrm{j}}=1$, the individual enterprise $\mathrm{j}$ obtains innovative coefficient $x=0.3$ after innovation; When node $j$ adopts innovative imitation strategies during the i-th game, $\mathrm{Yi}, \mathrm{j}=0$, the individual enterprise $\mathrm{j}$ obtains innovative coefficient $\mathrm{x}=0$ after innovation.

$\beta= \begin{cases}\mu / 1+\mu & Y_{i, j}=1 \\ (1-\mu)^{(1-\mu)} & Y_{i, j}=0\end{cases}$

Where, $\mathrm{u}$ is the ratio of innovators in the network group.

Moreover, based on $\mathrm{N} 0=a$, the paper assumes the following iterative relationship for innovative stock gains

$N_{1}=\left(N_{0}+S_{1}\right) / 2$

$N_{2}=\left(N_{0}+S_{1}+S_{2}\right) / 3$

$N_{3}=\left(S_{1}+S_{2}+S_{3}\right) / 3$

\subsection{Game Evolution: Two Standard Setting of Game Evolution}

Regard the average revenue of the surrounding neighborhood as the influence of strategy change, Assumption: in the $\mathrm{i}$-th round game, a single enterprise $\mathrm{j}$ makes policy changes: $Y_{i, j}: 1$ to 0 or 0 to 1 . When enterprise $j$ receives less than average earnings of the surrounding neighborhood in the i-th round of the game then $S_{\mathrm{i}, j}<\left(\sum_{0}^{k} S_{i, j, l}\right) / k$, individual enter- prise $\mathrm{j}$ makes a strategic adjustment. It imitates the same game strategy with neighbors. 1 represents the neighbor of node $\mathrm{j}$ and $\mathrm{k}$ is the number of its neighbors, $\mathrm{k}=(0,1,2,3,4$ …...).

Regarding the largest revenue earned amongst the neighborhood as the influence of strategy change. Assumption: when earnings enterprise $\mathrm{j}$ received in the $\mathrm{i}$-th round of the game is less than the earning that neighbor I received in the same round (I gained most among all the neighbors of $j$ ) that is, when $S_{i, j}<\max \left(S_{i, j, l}\right)$, individual enterprises $\mathrm{j}$ will make strategic adjustments, it will imitate the same strategy with I.

Strategy update process can be expressed as When $S_{i, j}<\max \left(S_{i, j, l}\right) \quad S_{i, j, l}$ Where, $\mathrm{S}_{\mathrm{i}, \mathrm{j}}$ represents the revenue that node $\mathrm{j}$ obtained in the $\mathrm{i}$-th round of the game, $\mathrm{S}_{\mathrm{i}, \mathrm{j}}, 1$ represents the revenue that $\mathrm{I}$, who is the neighbor of node $\mathrm{j}$, obtained in the $\mathrm{i}$-th round game and I obtained the most.

\subsection{E. The Initial Matrix Set of Imitation - Innovation}

During the simulation, the paper established random network, small-world networks and scale-free network structure made by the 20 nodes (node name: AT) set the "imitation-innovation" initial matrix $[1,0,0,1,0,0,0,1,0,0,0,0,0,0$, $1,0,0,0,1,0]$. The nodes $\mathrm{A}, \mathrm{D}, \mathrm{H}, \mathrm{O}, \mathrm{S}$ are initial innovators that are randomly selected, defined as "1"; remaining 15 are initial imitators, defined as "0."

\section{COMPARATIVE ANALYSIS REGARDING THE AVERAGE REVENUE OF THE SURROUN- DING NEIGHBORHOOD AS THE STANDARD OF STRATEGY}

\subsection{A. The Analysis of Simulation Results Under Ran- dom Cluster Network Structure}

Under random network structure, when individual enterprises treat the average revenue of the neighbors around as a policy updating standard, the simulation results as shown in Fig. (1).

As shown in Fig. (1), innovators ratio presents volatility and this volatility fluctuates from strong to weak and to the innovation ratio of 0.3 until disappearance. During the 1-3 round of the game, innovators presents rule number fluctuations of $0-5-0$; in the $4-9$ rounds, innovators presents rule number fluctuations of 5-0; in the 10-23 rounds, innovators presents rule number fluctuations of $5-4$; in the 24-33 rounds, innovators number remains unchanged at 5 , but main innovation presents "ADHOR-CJKQT" rule changes; in the 34-39 round, innovators presents rule number fluctuations of 6-5; from the 40 round, innovators number remain unchanged 6, but main innovation presents "ADHMOSCJKPQT" rule changes.

\subsection{B. The Analysis of Simulation Results Under Small- world Cluster Network Structure}

Under small-world network structure, When individual enterprises treat the average revenue of the neighbors as a 

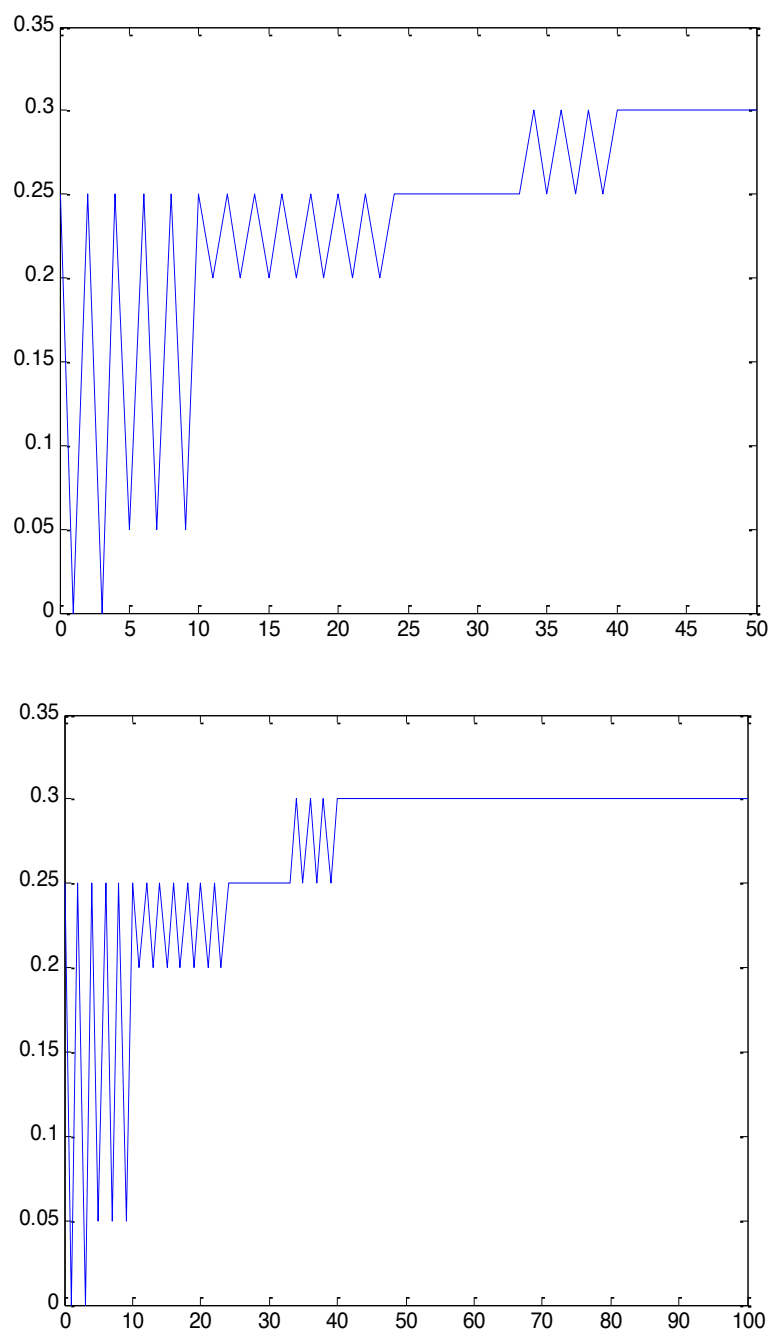

Fig. (1). Imitation - Innovative state evolution chart in random network structure, when regarding the average revenue of the surrounding neighborhood in last round as the standard of strategy changing (50/100).

policy updating standard, the simulation results are as shown in Fig. (2).

As shown in Fig. (2), innovators ratio presents volatility it fluctuation at about 0.3 . Fluctuations intensity decreases then gradually increase until it reaches the innovation cycle fluctuation ratio $0.1-0.4$. During the $1-5$ rounds of the game, innovators presents rule number fluctuations of 5-0; in the 67 rounds, innovators presents rule number fluctuations of 51 ; in the 8-17 rounds, innovators presents rule number fluctuations of $5-1$; in the $40-45$ rounds, innovators presents rule number fluctuations of 7-2; from the 46 rounds onwards, innovators number increases to 20 .

\subsection{The Analysis of Simulation Results Under Scale- free Networks}

In a scale-free network structure, when regarding the average revenue of the surrounding neighborhood in last round as the standard of strategy changing, the simulation results are shown in Fig. (3).

As shown in figure, innovation rate shows great fluctuations, and during the first six games, fluctuations becomes the largest and the difference is 0.25 , Then relatively small amplitude fluctuations erratically appear, innovation ratio of the entire group shows the regularity of the cycle fluctuations is about $0.3-0.2$ after the $28^{\text {th }}$ round. Specifically, in the 1-3 rounds, innovators presents rule number fluctuations of $0-5-0$; in the $4-5$ rounds, innovators presents rule number fluctuations of 5-1; in the 6-11 rounds, innovators presents rule number fluctuations of 5-3; the number of innovators remains stable 4 short during this stage, in the 15-20 rounds, innovators presents rule number fluctuations of 3-4; in the 21-22 rounds, the number of innovators remains stable 4 short; in the 23-27 rounds, innovators presents rule number fluctuations of 5-4; from the 28 round onward, innovators number increases to 20 and maintain a stable fluctuation.

\section{CONCLUSION AND FUTURE WORK}

Compared with the six figures above, we can see that when regarding the average revenue of the surrounding neighborhood as the standard of strategy change:

First, all in all, under three network clusters structure, enterprises have an increasing trend to balance innovation. But 

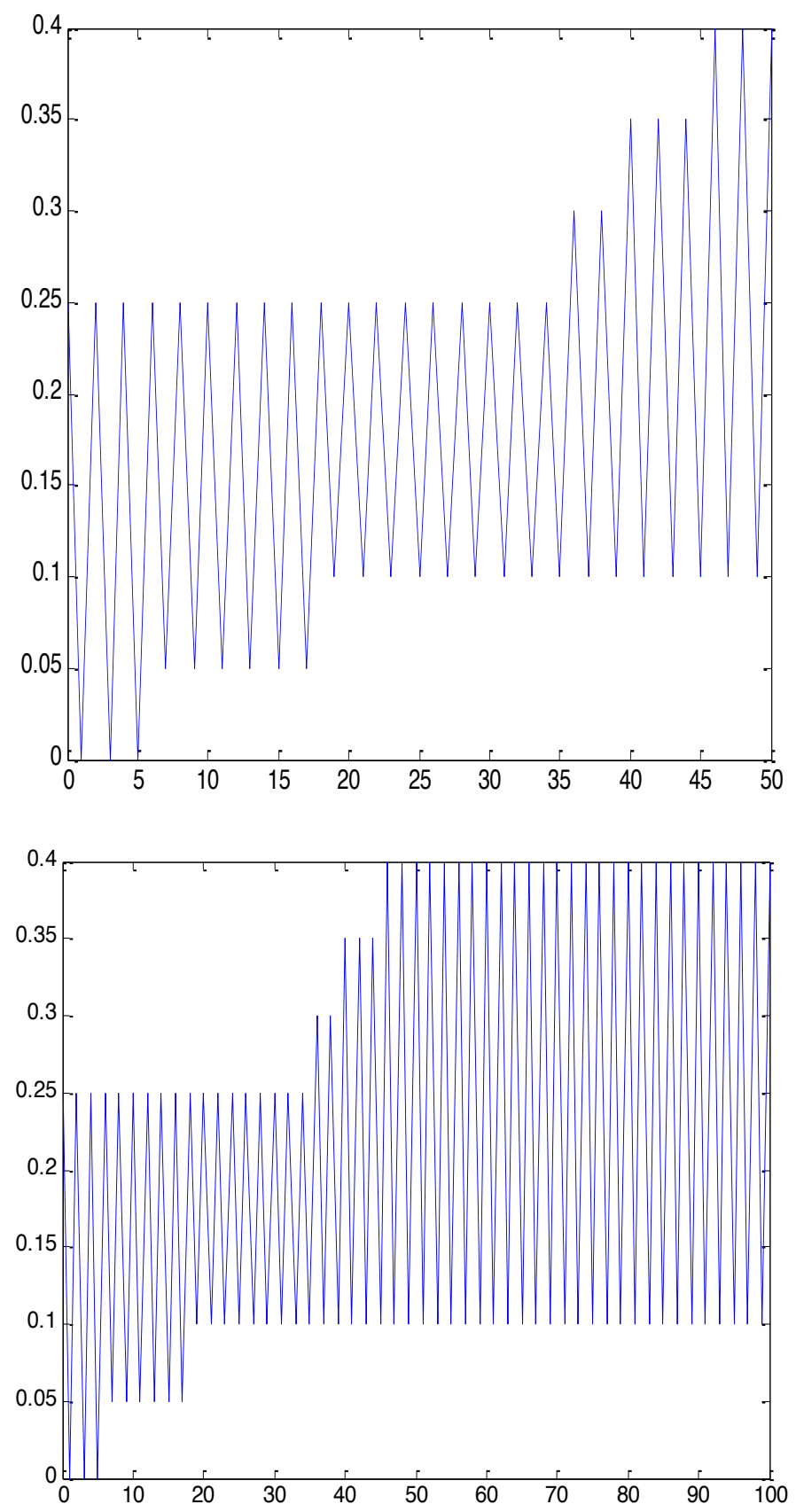

Fig. (2). Imitation - Innovative state evolution chart in small-world network structure, when regarding the average revenue of the surrounding neighborhood in last round as the standard of strategy change (50/100).

only under the random network cluster structure, enterprises will eventually achieve a stable equilibrium innovation (after 40 games, always keeping innovation ratio of 0.3 ), while under the small-world network structure and no-scale cluster structure, the results are opposite. That is, enterprises tend to achieve a state of fluctuant innovation ratio (after 45 games, the innovation rate under small-world network structure of the cluster is maintained at 0.1-0.4; after 28 games, the ratio scale cluster innovation network structure is kept under 0.2$0.3)$.

Second, under the three network clusters structure, the ratio of innovation demonstrates enormous fluctuations. Especially, the small-world network structure of the cluster, the difference is always over 0.15 . However, under random cluster network structure, the rate of innovation fluctuates reduces with the difference of first 0.25 and then gradually reduces further to reach the final state of stable equilibrium. Compared with the fluctuation ratio of random cluster innovation network structure, under no-scale cluster network structure, the ratio of innovation fluctuate from large to small, even to a briefly stable stage. Thereafter, after about 23 games, ascending trend is shown and finally maintains at a difference of about 0.1 .

Third, when regarding the average revenue of the surrounding neighborhood as the standard of individual enterprise's updating strategies, three cluster network structures 

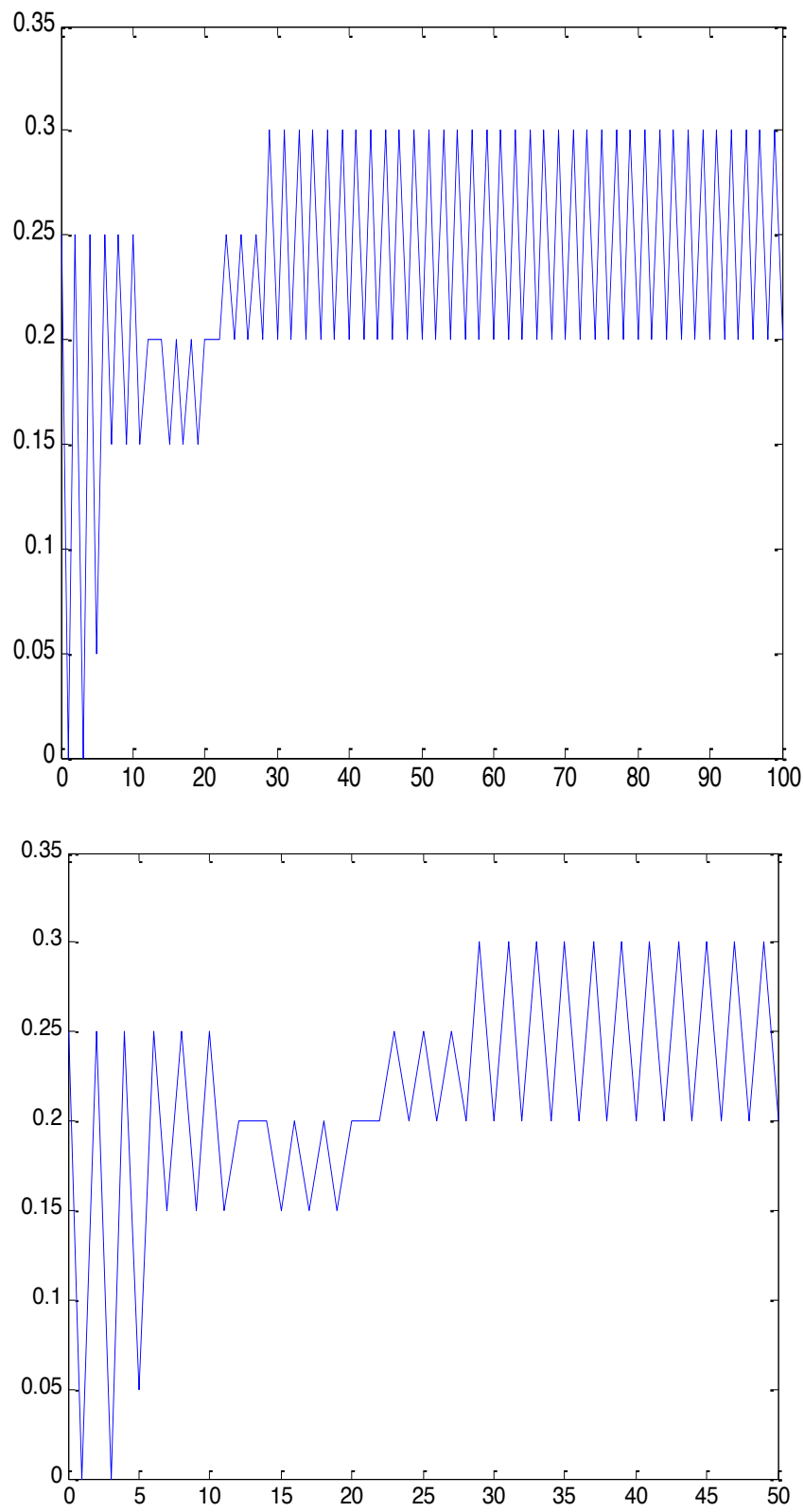

Fig. (3). Imitation - Innovative state evolution chart in scale-free network structure, when regarding the average revenue of the surrounding neighborhood in last round as the standard of strategy changing $(50 / 100)$.

can not achieve the equilibrium stage where all enterprises within the cluster are involved to innovate. The largest cluster innovation equilibrium ratio is 0.4 . In other words, the proportion of innovative groups within cluster would not exceed $40 \%$.

\section{CONFLICT OF INTEREST}

The authors confirm that this article content has no conflict of interest.

\section{ACKNOWLEDGEMENTS}

The authors wish to thank everyone who helped them. This study are subsidized by the "Zhijiang youth project" of
Zhejiang philosophy and social science planning, "a study on the impact of cluster network structure on cooperative R\&D Alliances" (13ZJQN022YB); the information economy and society-funded research projects of Development Research Center of Zhejiang Province (2013xxhjd004z); the research program of humanistic and social science from ministry of education "a study on the same trade joint $\mathrm{R}$ and $\mathrm{D}$ guide mechanism based on cooperation and competition game" (12YJC630202), key disciplines (business management) Graduate Innovation Program of Zhejiang province in school of Management of Hangzhou Dianzi University (GK120201204004/001); College of Humanities and Social Sciences provincial key research base to cultivate outstanding master's degree thesis project "Management Science and Engineering." (GK130202204004/018). The provincial key disciplines 
(business administration)high level academic papers breeding project of Hangzhou Dianzi university management school (GK130204204004).

\section{REFERENCES}

[1] A.L. Barabási, "Scale-free networks: a decade and beyond", Science, vol. 325, pp. 412-413, 2009.

[2] M.E.J. Newman, "Models of the small world", Journal of Statistical Physics, vol. 101, pp. 819-841, 2000.

[3] A.L. Barabási, and R. Albert, "Emergence of scaling in random networks", Science, vol. 286, pp. 509-512, 1999.

[4] X.F. Wang, and G. Chen, "Complex networks: small-world, scale-free and beyond", Circuits and Systems Magazine IEEE, vol. 3, pp. 6-20, 2003.

[5] B.Y.A.L. Barabási, and E. Bonabeau, "Scale-Free", Scientific American, 2003.
[6] D. Mahbuba, and R. Rousseau, "The Matthew effect and a relation with concept symbols and defaults", Annals of Library and Information Studies, vol.58, pp. 335-345, 2011.

[7] G.I. Allen, Z. Liu, "A local poisson graphical model for inferring networks from sequencing data", IEEE Trans Nanobioscience, 2013.

[8] H.S. Chen, "The Relationship between Technology Industrial Cluster and Innovation in Taiwan", Asia Pacific Management Review, vol. 16, pp. 277-288, 2011.

[9] J. Mason, "Tangible affinities and the real life fascination of kinship", Sociology, vol. 42, pp. 29-45, 2008.

[10] R.D. Dietz, "The estimation of neighborhood effects in the social sciences: An interdisciplinary approach", Social Science Research, vol.31, pp. 539-575, 2002.

[11] W.H. Davidson, "The location of foreign direct investment activity: country characteristics and experience effects", Journal of International Business Studies, 2011.

Received: September 22, 2014

(C) Wang and Pan; Licensee Bentham Open.

This is an open access article licensed under the terms of the Creative Commons Attribution Non-Commercial License (http://creativecommons.org/licenses/by-nc/3.0/) which permits unrestricted, non-commercial use, distribution and reproduction in any medium, provided the work is properly cited. 